\title{
The role of plasma-wall interactions in thermal instabilities at the tokamak edge
}

\author{
M. Z. Tokar and F. A. Kelly \\ Institut für Plasmaphysik, Forschungszentrum Jülich GmbH, Association FZJ-Euratom, \\ 52425 Jülich, Germany
}

(Received 13 May 2003; accepted 1 August 2003)

\begin{abstract}
Plasma-wall interaction leads to the release of impurities and neutrals of the working gas, which contribute significantly to the energy losses from the plasma edge, and therefore, crucially affects the development of thermal instabilities in fusion devices. An analytical model for impurity radiation is proposed, which takes into account the erosion mechanisms of wall material and the motion of impurity particles across magnetic surfaces. The temperature dependence of radiation losses is found to be very different from that predicted by the coronal approximation often used in considering thermal instabilities. The consequences for the development of poloidally symmetric detachment and multi-faceted asymmetric radiation from the edge (MARFE) are analyzed. It is demonstrated that the MARFE threshold principally depends on the mechanism by which working gas neutrals are released from the wall and on the neutral's properties, e.g., their ionization rate. The results of density limit experiments in Tokamak Experiment for Technology Oriented Research [Proceedings of the 16th IEEE Symposium on Fusion Engineering, 1995 (Institute for Electrical and Electronics Engineers, Piscataway, NJ, 1995), p. 470] and Joint European Torus [Rebut et al., Fusion Eng. Des. 22, 7 (1993)] are interpreted. (C) 2003 American Institute of Physics.
\end{abstract}

[DOI: $10.1063 / 1.1613963]$

\section{INTRODUCTION}

Thermal instabilities at the plasma edge can lead to the onset of plasma detachment and multi-faceted asymmetric radiation from the edge (MARFE) and, therefore, they are of importance for the fundamental problem of the density limit in fusion devices. ${ }^{1}$ In theoretical models for these instabilities, ${ }^{2-7}$ the energy losses due to excitation and ionization of hydrogen neutrals and impurities are normally included as important phenomena, however, the densities of these particles are often assumed as given. In reality, they are determined by the plasma fluxes to the walls, which are changing in the presence of perturbations of plasma parameters. In an instability analysis, this effect should be accounted for in the linearization of the transport equations. In addition, the pattern of plasma-wall interaction can be also of importance. Consider the MARFE, arising on the high field side (HFS) near the inner wall. In limiter tokamaks, this "wall" MARFE normally leads to the density limit. ${ }^{8-11} \mathrm{Re}$ cently, the wall MARFE was also observed in the divertor configuration in Joint European Torus (JET), where it occurs after the X-point MARFE and ultimately determines the maximum density in L-mode deuterium plasmas. ${ }^{12}$ As demonstrated earlier, ${ }^{7}$ unstable perturbations leading to the wall MARFE have their largest amplitude on the HFS. Thus, the proximity of the last closed magnetic surface (LCMS) to the wall at the HFS should be of great importance for the instability threshold.

The significance of plasma-wall contact at the HFS for MARFE onset was profoundly demonstrated in experiments on Tokamak Experiment for Technology Oriented Research (TEXTOR), ${ }^{13}$ where the critical density, $n_{\text {MARFE }}$, was nearly doubled by increasing the clearance between the LCMS and the inner wall. This occurred even though the impurity concentration and effective plasma charge were not reduced by this procedure. The reduction of the plasma contact with the wall at the HFS was balanced by a stronger impurity release from limiters at the low field side. ${ }^{11}$ Recently, similar results have been obtained on JET. ${ }^{14}$

By increasing the plasma wall clearance, the outflow of charged particles and energy to the inner wall is reduced and, consequently, the influx of recycling neutrals of the working gas and eroded impurities into the plasma is decreased. The properties of the recycling particles, e.g., their ionization rate, control their penetration into the plasma and thus should be taken into account in a MARFE analysis. This could, in particular, explain observations on JET that no wall MARFE occurred in helium plasmas and that a much higher density limit was achieved compared to deuterium plasmas. ${ }^{14}$

The influx of impurities is determined both by the plasma outflow to the wall and by the erosion rate of the wall material. Therefore, the erosion mechanisms should be also included in a realistic model of the MARFE. This is supported by the observation on TEXTOR ${ }^{15}$ that a higher $n_{\text {MARFE }}$ can be achieved with freshly boronized or siliconized walls even though the radiation characteristics are similar to those of carbon dominated discharges. At the same time, the erosion mechanisms of these impurities ${ }^{16}$ are different. This is especially true at the low plasma temperatures relevant to MARFE formation, where the release of carbon impurity is dominated by chemical sputtering. ${ }^{17,18}$ Also, the dependence of hydrogen absorption and recycling properties on the wall coating material ${ }^{19}$ can be of significant importance. 
In the present paper, an analytical model is proposed for the threshold of poloidally symmetric detachment and wall MARFE, which includes plasma-wall interaction. The next section describes a model for impurity radiation, which includes both the impurity release from the wall and the transport of impurity neutrals and ions across magnetic surfaces. It is shown that for a realistic level of the particle diffusion, the effective cooling rate of light impurities has a very weak temperature dependence in the temperature range relevant to MARFE formation. Constraints imposed by this fact on the development of radiative instabilities at the plasma edge are discussed in Sec. III. In Sec. IV, a model for the MARFE threshold is outlined. Discussion of the results and concluding remarks are given in Secs. V and VI.

\section{NONCORONA MODEL FOR IMPURITY RADIATION}

Many analytical models for radiative instabilities are based on the coronal equilibrium mode ${ }^{20}$ for impurity radiation, which neglects transport effects and assumes a balance between ionization and recombination processes. The impurity cooling rate $L_{I}$, i.e., the power radiated per one impurity particle and one electron, then depends only on the electron temperature $T$. The coronal $L_{I}, L_{c}$, has a strong maximum at a certain temperature, which is determined by atomic characteristics of the impurity.

At the plasma edge, however, the recombination of impurity particles released from the machine wall normally plays a minor role compared to the ionization and transport processes. ${ }^{21}$ An accurate calculation of the effect of impurity transport involves the solution of coupled transport equations for different charge states and requires the use of numerical codes. ${ }^{21-26}$ In order to make qualitative predictions, several approximate noncoronal approaches were put forward. In Refs. 27 and 28 it was proposed to shift the coronal temperature dependence of the cooling rate to higher temperatures, i.e., $L_{I}(T)=L_{c}(\zeta T)$, where $0.3<\zeta<1$. This approach is supported by the observation that impurities released from the wall diffuse into regions of high electron temperature before they can be strongly ionized, i.e., they remain in low ionized states with small excitation energies and high radiation efficiency. Therefore, the radiation losses are better represented by the coronal radiation rates of a lower temperature, which for typical impurities and edge conditions are generally higher.

A more quantitative estimate can be obtained by integrating impurity transport equations over the plasma radius to obtain zero-dimensional particle balance equations, which include a characteristic time of impurity residence in the plasma. ${ }^{29-31}$ As a result, the effective cooling rate becomes a function of the product of this time and electron density. The stronger the impurity transport, the smaller the residence time and the more significant the increase of the cooling rate compared to the coronal level should be expected. In some papers, ${ }^{31,32}$ an extraordinary importance of impurity chargeexchange with hydrogen neutrals, recycling from the wall, had been anticipated. Charge-exchange leads to the conversion of "dim" highly ionized particles into radiant ions of a low charge and, finally, to an enhancement of the cooling rate. However, a self-consistent one-dimensional treatment ${ }^{21}$ demonstrated that due to ionization the density of hydrogen neutrals decays too fast with increasing distance from the walls to be of importance in the region where sufficiently ionized impurity species are present. Henceforth, this process will be neglected.

In this section, we derive analytical expressions for the effective noncoronal cooling rate and radiation losses from light impurities which explicitly include the transport characteristics. It is assumed that (i) the plasma parameters, density $n$ and temperature $T$, are characterized by their mean values in the edge region where impurities mainly radiate; (ii) impurity ion transport is diffusive; (iii) recombination of impurity ions is negligibly small; and (iv) they can be separated into groups of "radiant" and "dim" charge states. The former are ions with excitation energies much less than their ionization potentials. In the case of light impurities such as $\mathrm{Be}, \mathrm{B}$, and $\mathrm{C}$, this group embraces the ions of low charges up to that of Li-like ones. ${ }^{33}$ The latter includes $\mathrm{He}$ and $\mathrm{H}$-like ions with very high excitation energies, and fully stripped impurity nuclei.

Impurity particles sputtered from the wall enter the plasma volume as neutrals. Due to ionization, their density decays exponentially with the distance $x$ toward the plasma core: $^{34}$

$$
n_{0}=\frac{j_{0}}{V_{0}} \exp \left(-\frac{x}{l_{0}}\right),
$$

where $j_{0}$ and $V_{0}$ are the influx density and velocity of impurity neutrals, $l_{0}=V_{0} / k_{\text {ion }}^{0} n$ is their penetration depth with $k_{\text {ion }}^{0}$ being the ionization rate coefficient.

The total density of impurity ions of all charged states, $n_{I}$, is governed by the equation

$$
-D_{\perp}^{I} \frac{d^{2} n_{I}}{d x^{2}}=k_{\text {ion }}^{0} n n_{0},
$$

where the diffusivity $D_{\perp}^{I}$ is assumed to be the same for all charges. Equations (1) and (2) together with the boundary conditions $n_{I}(x=0)=0$ and $\left(d n_{I} / d x\right)\left(x \gg l_{0}\right)=0$, which assume that the impurity ions are effectively absorbed in the scrape-off layer beyond the LCMS and only sourced at the wall, yield ${ }^{34}$

$$
n_{I}=\frac{j_{0} l_{0}}{D_{\perp}^{I}} \times\left[1-\exp \left(-\frac{x}{l_{0}}\right)\right] .
$$

Radiant impurity ion species with charges up to that of the Li-like particles, $Z_{\mathrm{Li}}$, have roughly the same cooling rate, $L_{\text {rad }} \cdot{ }^{33}$ The total density of these particles, $n_{\text {rad }}=\Sigma_{Z=1}^{Z_{\mathrm{Li}}} n_{Z}$, is determined by the sum of the continuity equations for all charges up to $Z_{\mathrm{Li}}$. According to one-dimensional transport modelling, ${ }^{21}$ the Li-like particles make the dominant contribution to $n_{\text {rad }}$ in the region where they are ionized into Helike ions, and we may write the following equation for $n_{\text {rad }}$ :

$$
-D_{\perp}^{I} \frac{d^{2} n_{\mathrm{rad}}}{d x^{2}}=k_{\mathrm{ion}}^{0} n n_{0}-k_{\mathrm{ion}}^{\mathrm{Li}} n n_{\mathrm{rad}} .
$$

The first term on the right-hand side is the source due to ionization of neutrals and the second one is the sink due to 


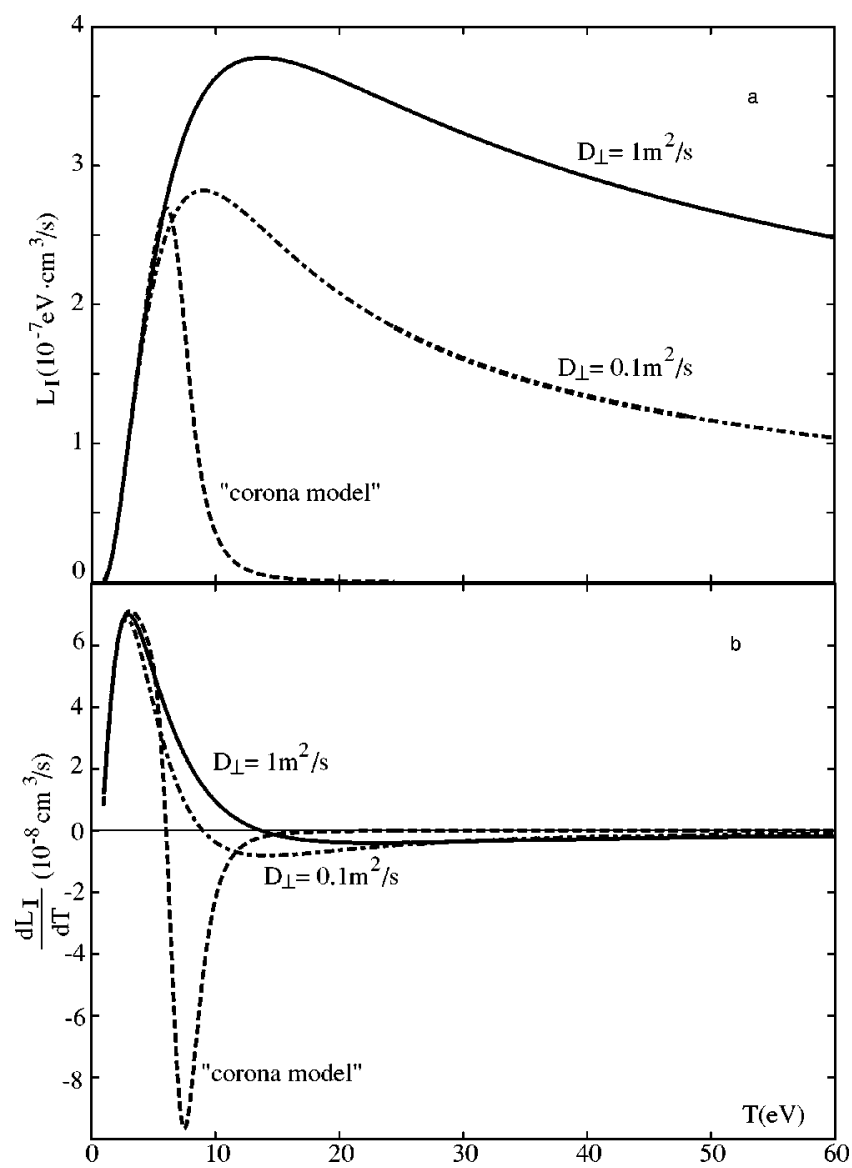

FIG. 1. The temperature dependence of carbon's cooling rate and its derivative computed according to Eq. (7) in corona approximation and for different magnitudes of impurity ion diffusion.

ionization into the He-like state. With the same boundary conditions as for the total impurity density, one obtains

$$
n_{\mathrm{rad}}=\frac{j_{0} l_{0}}{D_{\perp}^{I}} \frac{\alpha^{2}}{\alpha^{2}-1}\left[\exp \left(-\frac{x}{l_{\mathrm{rad}}}\right)-\exp \left(-\frac{x}{l_{0}}\right)\right],
$$

where $\alpha=l_{\text {rad }} / l_{0}$ and $l_{\text {rad }}=\sqrt{D_{\perp}^{I} / k_{\text {ion }}^{\mathrm{Li}} n}$.

The condition $d n_{\text {rad }} / d x=0$ determines the position of the maximum of $n_{\mathrm{rad}}: x_{\max }=l_{\mathrm{rad}}(\ln \alpha) /(\alpha-1)$. For the maximum value itself, we get

$$
n_{\mathrm{rad}}^{\max }=\frac{j_{0} l_{0}}{D_{\perp}^{I}} \frac{\alpha^{2}}{\alpha^{2}-1}\left(\alpha^{-1 /(\alpha-1)}-\alpha^{-\alpha /(\alpha-1)}\right) .
$$

The location where $n_{\text {rad }}$ and, thus, the radiation density, $n n_{\text {rad }} L_{\text {rad }}$, approach a maximum is the most probable location for the development of radiative instabilities. Therefore the cooling rate, which should be used in the instability criterion, $L_{I}$, is determined from the relation $n n_{\mathrm{rad}}^{\max } L_{\mathrm{rad}}$ $=n n_{I}\left(x_{\max }\right) L_{I}$ :

$$
L_{I}=L_{\mathrm{rad}} \frac{\alpha^{2}}{(\alpha+1)\left(\alpha^{\alpha /(\alpha-1)}-1\right)} .
$$

Figure 1(a) shows the temperature dependence of $L_{I}$ for carbon impurity computed for $n=2 \times 10^{13} \mathrm{~cm}^{-3}$ and different magnitudes of the ion diffusivity $D_{\perp}^{I}$. It was taken into account that according to the measurements ${ }^{17}$ the neutral car-

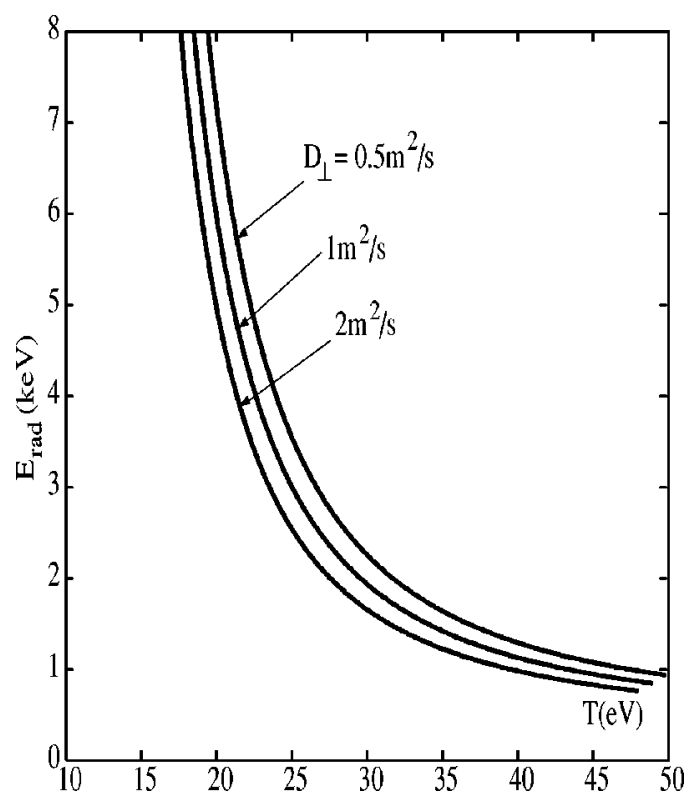

FIG. 2. Radiation potential of carbon and oxygen mixture in deuterium plasma with 2:1 ratio of impurity influxes.

bon velocity, $V_{0}$, reduces from $1.5 \times 10^{6} \mathrm{~cm} / \mathrm{s}$ at $T \gtrsim 20 \mathrm{eV}$ to $0.5 \times 10^{6} \mathrm{~cm} / \mathrm{s}$ when $T \lesssim 10 \mathrm{eV}$ due to the transition from physical sputtering to chemical erosion of carbon particles. The noncoronal model results are compared with the temperature dependence of the cooling rate in coronal equilibrium. For temperatures higher than $10 \mathrm{eV}$, where the recombination rate of He-like ions cannot compete with the ionization of Li-like ones, the coronal cooling rate is by several orders of magnitude lower than that found when impurity diffusion is taken into account. Thus for temperatures in the range of 20-60 eV where radiative instabilities, e.g., the MARFE, normally develop, ${ }^{11,35-37}$ the impurity density and, thus, radiation losses are determined principally by the competition between transport and ionization processes.

The radiating capability of an impurity is often characterized by its radiation potential, $E_{\text {rad }}$, defined as the total energy radiated per impurity particle during its confinement time in the plasma: ${ }^{35} \quad E_{\mathrm{rad}} \equiv q_{\mathrm{rad}} / j_{0}$ with $q_{\mathrm{rad}}$ $=\int_{0}^{\infty} n n_{\mathrm{rad}} L_{\mathrm{rad}} d x$. By using Eq. (5) one finds

$$
E_{\mathrm{rad}}=\frac{L_{\mathrm{rad}}}{k_{\mathrm{ion}}^{\mathrm{Li}}} \frac{l_{0}}{l_{\mathrm{rad}}+l_{0}} .
$$

Figure 2 shows the temperature dependence of the effective impurity radiation potential computed with different values of $D_{\perp}^{I}$ for TEXTOR ohmic plasmas where carbon and oxygen are the dominant impurities. In this case,

$$
E_{\mathrm{rad}}=\frac{E_{\mathrm{rad}}^{\mathrm{C}}+\alpha_{\mathrm{O}} \times E_{\mathrm{rad}}^{\mathrm{O}}}{1+\alpha_{\mathrm{O}}},
$$

where $E_{\mathrm{rad}}^{\mathrm{C}, \mathrm{O}}$ are the radiation potentials of $\mathrm{C}$ and $\mathrm{O}$ impurity species and a ratio of oxygen to carbon flux, $\alpha_{\mathrm{O}}$, of 0.5 was assumed. ${ }^{35}$ This dependence is in agreement with the experimentally measured one. ${ }^{35}$ 


\section{EDGE RADIATIVE INSTABILITY IN THE NONCORONAL APPROXIMATION}

From the point of view of plasma thermal stability not only the absolute level of radiation losses but also their temperature dependence and, in particular, the derivative $d L_{I} / d T$, shown in Fig. 1(b), are of importance. In the corona approximation, $d L_{I} / d T$ for carbon has a large negative value in a temperature range near $10 \mathrm{eV}$. Such a behavior is often considered to be the main cause for radiative instabilities at the plasma edge in fusion devices: a spontaneous reduction of the temperature leads to an increase of $L_{I}$ and radiation losses and the temperature drops further. In the temperature range where $d L_{I} / d T$ is negative its absolute value is decreased by an order of magnitude by going from the corona case to a particle diffusivity of $1 \mathrm{~m}^{2} / \mathrm{s}$ typical at the plasma edge in fusion devices. ${ }^{38}$ Therefore, the radiative instability due to the temperature dependence of the cooling rate can develop at a plasma density much higher than the experimental one. ${ }^{35}$ The same conclusion was drawn by considering the response of radiation losses to small temperature perturbations. $^{39}$

This does not mean that impurity radiation cannot trigger a thermal instability at the plasma edge. However, the instability scenario is different from the expected one. In the case where $d L_{I} / d T$ is responsible for an instability, the radiation density increases and temperature drops locally. If this mechanism is not efficient, an instability can still develop if a reduction in the temperature leads to a widening of the radiation layer and an increase of the integrated energy losses from the plasma. ${ }^{40}$ Such a widening occurs since the ionization rate of radiative ions diminishes with decreasing temperature and they penetrate deeper into the plasma.

In this section, we consider poloidally homogeneous perturbations of the edge plasma temperature, which should lead to a radial detachment. ${ }^{35}$ In order to determine under which conditions such perturbations become unstable, we proceed from the power balance of the plasma edge, where radiation of light impurities and ionization of recycling hydrogen neutrals are localized:

$$
\frac{d E}{d t}=S_{0}\left(q_{\text {core }}-q_{\text {loss }}\right) .
$$

Here $E$ is the thermal energy stored in this plasma region, $S_{0}$ is the LCMS area, $q_{\text {core }}$ is the density of the heat flux from the plasma core and $q_{\text {loss }}$ the power flux density lost due to impurity radiation, plasma convection, and conduction, and spent on ionization and excitation of recycling hydrogen neutrals. The first contribution, $q_{\text {rad }}$ has been computed in the preceding section. The convected energy losses are $3 T_{L} \Gamma_{p}$, where $T_{L}$ and $\Gamma_{p}$ are the plasma temperature and charged particle flux density at the LCMS. According to onedimensional modelling, ${ }^{21,41} T_{L}$ is by a factor $\tau$ of 2 lower than the averaged temperature in the edge, $T$. For a polynomial dependence of the particle diffusivity on the plasma parameters, $\quad D_{\perp} \sim n^{\eta} T^{\theta}, \quad$ one has ${ }^{41} \quad \Gamma_{p}=\left[D_{\perp} /(\eta\right.$ $+2)] n^{2} \sigma_{*}$. Here, the characteristic cross section for attenuation of recycling neutrals, $\sigma_{*}$, is determined by the rate coefficients for hydrogen ionization and charge exchange, $k_{\text {ion }}^{H}$ and $k_{\mathrm{cx}}^{H}: \sigma_{*} \approx \sqrt{k_{\text {ion }}^{H}\left(k_{\text {ion }}^{H}+k_{\mathrm{cx}}^{H}\right)\left(m_{H} / T\right)}$. The power lost in the interactions of charged particles with neutrals is equal to $\Gamma_{p} E_{i}$ with $E_{i}$ being the difference of the energy lost by electrons on ionization and excitation processes ${ }^{42}$ and the kinetic energy of neutrals gained by ions. The energy loss on conduction is determined by the perpendicular heat conductivity, $\kappa_{\perp}$, and the temperature $e$-folding length at the LCMS, $\delta_{T}$. Finally, we obtain

$$
q_{\mathrm{loss}}=\Gamma_{p}\left(3 T_{L}+E_{i}+Y E_{\mathrm{rad}}\right)+\kappa_{\perp} \frac{T_{L}}{\delta_{T}},
$$

where the impurity erosion yield, $Y \equiv j_{0} / \Gamma_{p}$, is introduced.

The thermal energy in the edge plasma changes with the temperature. Consider small perturbations, $\widetilde{E} \sim \widetilde{T} \sim \exp (\gamma t)$. Instabilities at the plasma edge develop fast compared to the processes in the plasma core, which determine $q_{\text {core }}$, and henceforth this will be assumed constant. By linearizing Eq. (9) with respect to $\widetilde{T}$, one obtains for the growth rate of perturbations:

$$
\gamma \sim-\frac{\partial q_{\text {loss }}}{\partial T}
$$

Thus, the stability of stationary states is determined by the sign of the derivative of the total energy losses with respect to the edge temperature. Qualitatively, the criterion is if $\partial q_{\text {loss }} / \partial T<0$ a spontaneous reduction of $T$ would lead to an increase in the energy losses and a further reduction of the temperature. For unstable states, the increase in the total energy losses occurs in spite of reduction in the convection and conduction contributions and is caused by the increasing impurity radiation potential. The latter is due to the growing width of the radiative layer since radiating ions penetrate deeper into the plasma when their ionization rate drops with decreasing temperature.

This "energetic" approach gives the same instability criterion as a rigorous eigenvalue analysis, at least in the cases when such an analysis was performed by analytical means. ${ }^{40}$ For the problem in question, this analysis was first done in Ref. 3 where only radiation and conduction losses were taken into account and the "box" model was adopted for the impurity cooling rate: $L_{I}\left(T \leqslant T_{\max }\right)=$ const, $L_{I}\left(T>T_{\max }\right)=0$. Assuming $T_{L} \equiv 0$, it was demonstrated that no instability is possible at a radiative fraction $\gamma_{\text {rad }}$ less than 1 unless a finite length of perturbations along magnetic field lines, is implied. This conclusion was disputed, however, in Ref. 39 where a finite $e$-folding temperature length at the LCMS, $\delta_{T}$, and convective energy losses were taken into account. It was shown that in this case a poloidally symmetric radiative instability can occur at a $\gamma_{\text {rad }}$ significantly less than 1 in agreement with observations. ${ }^{35}$ Unfortunately, for the noncorona radiation model discussed in the present paper an analytical eigenvalue analysis is impossible. However, the results based on the analysis of $q_{\text {loss }}$ temperature dependence are in agreement with numerical self-consistent modelling. ${ }^{21}$

Figure 3(a) demonstrates the temperature dependence of $q_{\text {loss }}$ for different plasma densities computed for parameters typical in ohmic TEXTOR plasmas: ${ }^{35} D_{\perp}=1 \mathrm{~m}^{2} / \mathrm{s}, \kappa_{\perp}=2$ $\times 10^{19} \mathrm{~m}^{-1} \mathrm{~s}^{-1}, \delta_{T}=5 \mathrm{~cm}$. The carbon erosion coefficient 


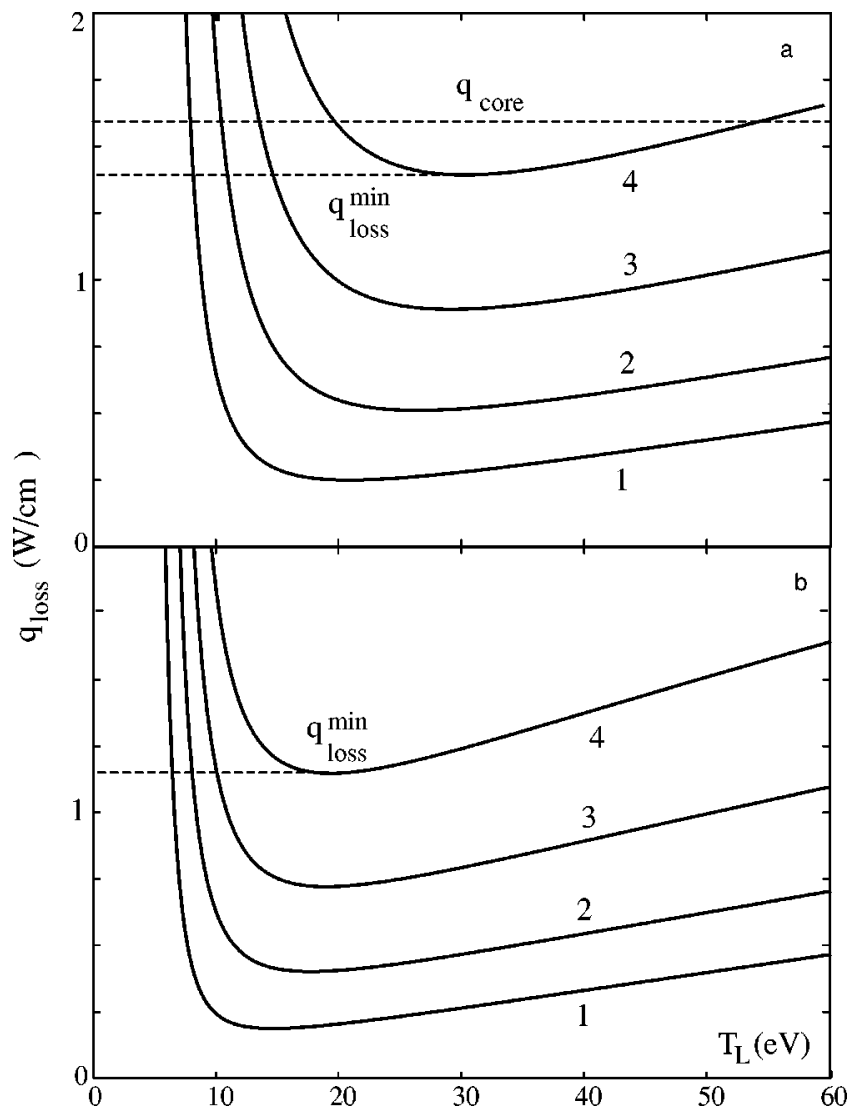

FIG. 3. The density of energy losses from the plasma surrounded by carbonized (a) and boronized (b) walls for different magnitudes of the plasma density: (1) $10^{13} \mathrm{~cm}^{-3}$, (2) $2 \times 10^{13} \mathrm{~cm}^{-3}$, (3) $3 \times 10^{13} \mathrm{~cm}^{-3}$, and (4) 4 $\times 10^{13} \mathrm{~cm}^{-3}$

$Y_{\mathrm{C}}$ is the sum of the physical sputtering rate, which is sensitive to the plasma temperature, ${ }^{16}$ and that of chemical erosion, ${ }^{18}$ depending weakly on $T$. By taking into account the release of oxygen (see Sec. II) the effective impurity erosion coefficient is given with $Y=Y_{\mathrm{C}} \times\left(1+\alpha_{\mathrm{O}}\right)$.

In stationary states, the plasma temperature at the edge is determined by the equality $q_{\text {core }}=q_{\text {loss }}$. If the plasma density is sufficiently low and the minimum of the energy losses, $q_{\text {loss }}^{\min }$, is smaller than $q_{\text {core }}$ two stationary states are possible but only the high temperature state and with $\partial q_{\text {loss }} / \partial T>0$ is stable. When, with increasing plasma density, $q_{\text {loss }}^{\min }$ exceeds $q_{\text {core }}$ no stationary state exists in the framework of the present model, the plasma edge cools down and radial detachment occurs.

In the case of wall boronization, a noticeable reduction of carbon and oxygen influxes both in ohmic and in auxiliary heated plasmas has been reported from many tokamaks. ${ }^{11,19,43-48}$ The degree of reduction varies, however, strongly from machine to machine, from 2 to 9 for carbon and from 3 to 15 for oxygen, depending on particular conditions, e.g., the gas composition used for boronization, the quality of covering produced, etc. Henceforth, we assume in our calculation that the erosion yields $Y_{\mathrm{C}}$ and $Y_{\mathrm{O}}$ are reduced after boronization by an averaged factor of 5 , however, the results do not noticeably change if the reduction factor is 4 or larger. The total impurity content is not significantly changed since boron physical sputtering is noticeably larger than that of carbon. In spite of this, the critical density for detachment in ohmic plasmas was increased by $20-30 \%$ after boronization. ${ }^{43}$ This is due to the fact that the level of boron chemical erosion is 1-2 orders of magnitude smaller than for graphite. ${ }^{18}$ Figure 3(b) shows $q_{\text {loss }}$ versus $T$ found in the case of boronized walls. In this case the increase of the radiation potential with decreasing plasma temperature (see Fig. 2), which leads to the minimum in $q_{\text {loss }}(T)$ dependence, is essentially compensated by the decrease of the boron physical sputtering yield with approaching to the threshold. Therefore, for the same plasma density $q_{\text {loss }}^{\min }$ is smaller compared to carbon and oxygen dominated plasmas [see Fig. 3(a)]. This means that if the plasma current and heating power are the same, the critical density for poloidally symmetric detachment should be higher, roughly by $20-25 \%$.

\section{MARFE THRESHOLD}

The density limit in auxiliary heated discharges is normally due to the development of poloidally asymmetric MARFE. ${ }^{11}$ In this paper, we consider only the case of wall MARFE developing in L-mode plasmas at the high field side. In order to analyze the importance of plasma-wall interaction for MARFE threshold, we proceed from the following equations for the particle and energy transfer in the plasma edge region by taking into account the transport both across magnetic surfaces ( $\perp$ direction) and along field lines ( $l$ direction):

$\nabla_{\perp} \Gamma_{\mathrm{H}}=-k_{\text {ion }}^{\mathrm{H}} n n_{\mathrm{H}}$,

$\frac{\partial n}{\partial t}+\frac{\partial n V}{\partial l}+\nabla_{\perp} \Gamma_{\perp}=k_{\mathrm{ion}}^{\mathrm{H}} n n_{\mathrm{H}}$

$3 \frac{\partial n T}{\partial t}+\nabla_{\perp} q_{\perp}+\frac{\partial}{\partial l}\left(-\kappa_{\|} \frac{\partial T}{\partial l}+5 n V T\right)$

$$
=-k_{\mathrm{ion}}^{\mathrm{H}} n n_{\mathrm{H}} E_{i}-n n_{\mathrm{rad}} L_{I},
$$

where $n_{\mathrm{H}}$ and $\vec{\Gamma}_{\mathrm{H}}$ are the densities of hydrogen neutrals and their flux, $V$ is the parallel plasma velocity, $\Gamma_{\perp}=-D_{\perp} \nabla_{\perp} n$ and $q_{\perp}=-\kappa_{\perp} \nabla_{\perp} T+3 \Gamma_{\perp} T$ are the perpendicular components of the plasma particle and heat flux densities, respectively, and $\kappa_{\|}$is the parallel electron heat conductivity.

In order to reduce the problem to one dimension the relations $\nabla_{\perp} \Gamma_{\mathrm{H}} \approx-\Gamma_{n} / \delta_{\text {edge }}$ and $\nabla_{\perp} \Gamma_{\perp} \approx \Gamma_{p} / \delta_{\text {edge }}$ will be used in Eqs. (12) and (13). Here, $\Gamma_{n}$ is the influx of neutrals into the plasma through the LCMS and $\delta_{\text {edge }} \approx 1 / n \sigma_{*}$ is the characteristic width of the edge recycling region. ${ }^{41}$ For stationary states discussed in the preceding section the condition of full particle recycling at the LCMS, $\Gamma_{P}=\Gamma_{n}$, is fulfilled. In considering fast perturbations leading to MARFE, one should take into account that $\Gamma_{n}$ is determined by the release of neutral particles from the wall and the time constant of this process is essentially controlled by the wall material properties. ${ }^{49}$ Here, we use a very simple approach to estimate the perturbation of $\Gamma_{n}$, which nevertheless allows to take into account the role of the wall. Following Ref. 51,

$$
\Gamma_{n}=\Gamma_{p} R_{P}+\mu c_{W},
$$


where the first contribution is from the ion reflection with the coefficient $R_{P}$ and the second one is due to desorption of neutrals from the wall with a rate coefficient $\mu$. The surface density of neutrals trapped in the wall, $c_{W}$, is governed by the balance equation ${ }^{50}$

$$
\frac{d c_{W}}{d t}=\Gamma_{p}-\Gamma_{n}=\Gamma_{p}\left(1-R_{P}\right)-\mu c_{W} .
$$

Consider small perturbations of plasma parameters proportional to $\exp (\gamma t) \times \Theta(l)$ near their stationary values. Close to the instability threshold, where $\gamma \rightarrow 0$, the pressure is maintained constant on magnetic surfaces with an accuracy of $\gamma^{2}$ (see, e.g., Ref. 7), and $n / n \approx-T / T$. By linearizing Eqs. (12), (13), (15), and (16) we find the relations

$$
\frac{\widetilde{\Gamma}_{p}}{\Gamma_{p}}=-\xi_{p} \frac{\widetilde{T}}{T}, \quad \frac{\widetilde{\Gamma}_{n}}{\Gamma_{p}}=-\xi_{w} \xi_{p} \frac{\widetilde{T}}{T}
$$

with

$$
\xi_{p}=\frac{2+\eta-\theta-\frac{d \ln \sigma_{*}}{d \ln T}}{2-\xi_{w}}
$$

and

$$
\xi_{w}=\frac{\gamma R_{P}+\mu}{\gamma+\mu} .
$$

Equation (14) is integrated over the edge region width with the boundary conditions at the LCMS, $d T / d x=T / \delta_{T}$, and at the interface with plasma core, $q_{\perp}=q_{\text {core }}$. The result is linearized for toroidally symmetric perturbations, for which $\partial l=q R \partial \vartheta$ where $\vartheta$ is the poloidal angle measured from the HFS, $q$ is the safety factor and $R$ is the major radius. This yields an equation for the poloidal variation of the temperature perturbation:

$$
\left(5 n \gamma \Theta-\frac{\kappa_{\|}}{q^{2} R^{2}} \frac{d^{2} \Theta}{d \vartheta^{2}}\right) \delta_{\text {edge }}=-\widetilde{q}_{\text {loss }}^{s} .
$$

Here, $q_{\text {loss }}^{s}=\Gamma_{p}\left(3 T_{L}+E_{i}+Y E_{\mathrm{rad}}\right)+g^{r r} \kappa_{\perp}\left(T_{L} / \delta_{T}\right)$ is nearly the same as $q_{\text {loss }}$ given by Eq. (10). The single difference is the presence in the conductive heat loss of the metric coefficient $g^{r r}$, which accounts for the magnetic geometry. Following Refs. 7 and 51, we consider cylindrical magnetic flux surfaces which are nonconcentric due to Shafranov shift $\Delta$ of their axes. In this case, $g^{r r}=1-\Delta_{1} \cos \vartheta$ and $\Delta_{1}$ $=2(d \Delta / d x) \ll 1$. Qualitatively, $g^{r r}$ reflects the variation of the distance between two magnetic surfaces with $\vartheta$. This distance approaches its maximum and the radial temperature gradient its minimum at the HFS, where $\vartheta=0$. It is important to notice that in the lowest order with respect to $\Delta_{1}$ the part of $q_{\text {loss }}^{s}$ proportional to the particle flux is $\vartheta$-independent. Physically, this follows from the fact that the density gradient and, thus, $\Gamma_{p}$, are governed by the penetration depth of neutrals whose motion is not influenced by the magnetic field. By using relations (17) and assuming $D_{\perp}^{I}$ $\sim n^{\eta_{I}} T^{\theta_{I}}$ and $\kappa_{\perp} \sim n^{\varepsilon} T^{\varsigma}$ we obtain

$$
\widetilde{q}_{\text {loss }}^{s}=\left(g^{r r} \kappa_{\perp} \frac{1+\varsigma-\varepsilon}{\tau \delta_{T}}-\Gamma_{p} \frac{E_{*}}{T}\right) \widetilde{T},
$$

where $E_{*}=3 T_{L} \phi_{T}+E_{i} \phi_{i}+Y E_{\mathrm{rad}} \phi_{\mathrm{rad}}$ with $\phi_{T}=\xi_{p}-1$,

$$
\phi_{i}=\xi_{w} \xi_{p}-\frac{d \ln E_{i}}{d \ln T}+\frac{d \ln E_{i}}{d \ln n}
$$

and

$$
\begin{aligned}
\phi_{\mathrm{rad}}= & \xi_{p}+\frac{d \ln \left(\frac{k_{\mathrm{ion}}^{L i}}{Y L_{\mathrm{rad}}}\right)}{d \ln T} \\
& +\frac{\alpha}{\alpha+1}\left[\frac{d \ln \left(\frac{k_{\mathrm{ion}}^{0}}{V_{0} \sqrt{k_{\mathrm{ion}}^{\mathrm{Li}}}}\right)}{d \ln T}-\frac{1-\theta_{I}+\eta_{I}}{2}\right] .
\end{aligned}
$$

Equation (18) reduces to the canonical form of Mathieu's equation $^{52}$

$$
\frac{d^{2} \Theta}{d z^{2}}+[a-2 p \cos (2 z)] \times \Theta=0
$$

with the independent variable $z=(\pi-\vartheta) / 2$ and the coefficients

$$
\begin{aligned}
& a=\frac{4 q^{2} R^{2}}{\kappa_{\|}}\left(\frac{\Gamma_{p}}{\delta_{\text {edge }}} \frac{E_{*}}{T}-\kappa_{\perp} \frac{1+\varsigma-\varepsilon}{\tau \delta_{\text {edge }} \delta_{T}}-5 n \gamma\right), \\
& p=\frac{2 q^{2} R^{2}}{\kappa_{\|}} \Delta_{1} \kappa_{\perp} \frac{1+\varsigma-\varepsilon}{\tau \delta_{\text {edge }} \delta_{T}} .
\end{aligned}
$$

As shown in Ref. 7, the eigenfunction of Eq. (21) with the largest growth rate $\gamma$, which satisfies the necessary periodic conditions is the even Mathieu function of the zero order, $c e_{0}$. This function has a maximum at $\vartheta=0$, i.e., reproduces the structure of a developed MARFE (see Fig. 1 in Ref. 7). There is an unique relation between the eigenvalues $a$ and $p$ and with an accuracy of $10 \%, a \approx-2 p /[1$ $+(4 / p)]$. This, together with Eq. (22), yields the growth rate of the perturbations:

$$
\gamma \approx \frac{1}{5 n \delta_{\text {edge }}}\left[\frac{\Gamma_{p} E_{*}}{T}-\frac{\kappa_{\perp}}{\tau \delta_{T}}(1+\varsigma-\varepsilon) \xi_{\Delta}\right],
$$

where

$$
\xi_{\Delta}=1-\frac{\Delta_{1}^{2}}{\Delta_{1}+\frac{2}{1+\varsigma-\varepsilon} \frac{\tau \delta_{\text {edge }} \delta_{T} \kappa_{\|}}{q^{2} R^{2} \kappa_{\perp} \tau} .}
$$

The critical condition corresponds to the perturbations just becoming unstable, i.e., $\gamma=0$, and can be used to write a relation for the critical plasma density. For the case discussed above with $D_{\perp}^{I}, D_{\perp}$, and $\kappa_{\perp}$ independent of the plasma parameters, we get

$$
n_{c r}=n_{\star} \sqrt{\frac{\xi_{\Delta} T}{E_{*}}},
$$

where $n_{\star}=\sqrt{2 \kappa_{\perp} \tau / D_{\perp} \delta_{T} \sigma_{*}}$. Here, the plasma temperature is not known and should be determined from the stationary plasma heat balance: $q_{\text {core }}=\left\langle q_{\text {loss }}^{s}\right\rangle \equiv q_{\text {loss }}$, where $\langle\cdots\rangle$ means the value averaged over the magnetic surface. This balance can be rewritten as a relation between the stationary values of the plasma density and temperature for a given $q_{\text {core }}$ : 


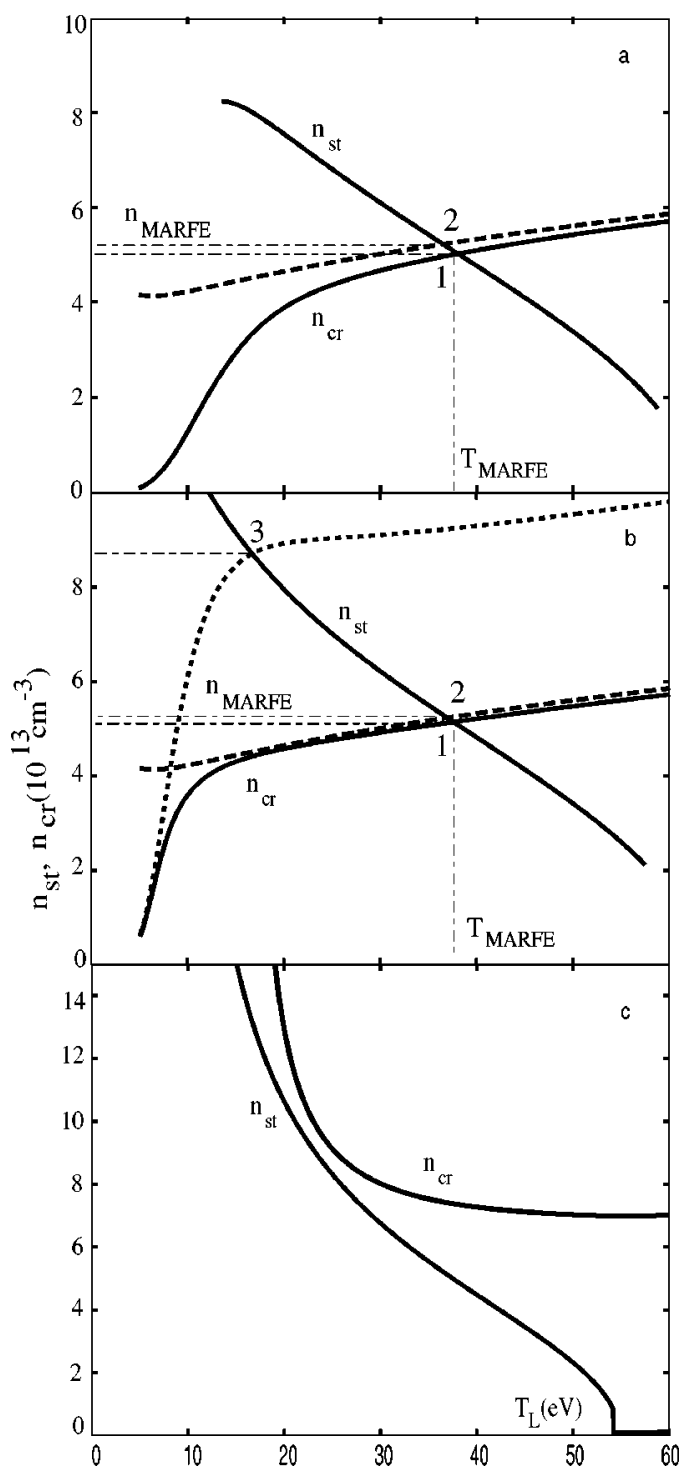

FIG. 4. Temperature dependences of the edge plasma density corresponding to stationary states and MARFE threshold for deuterium plasmas surrounded by (a) carbonized and (b) boronized walls in TEXTOR, and (c) for helium plasmas in JET. Points of intersection, 1-3, give the plasma parameters at the MARFE onset under different assumptions about the contribution of impurity radiation and particle recycling to the energy losses.

$$
n_{s t}=n_{\star} \sqrt{\frac{\frac{q_{\mathrm{core}} \delta_{T}}{\kappa_{\perp} \tau}-T}{3 \tau T+E_{i}+Y E_{\mathrm{rad}}}} .
$$

Equations (24) and (25) are transcendent equations for $n_{c r}$ and $n_{s t}$, which can be solved numerically by iteration. The plasma parameters at which the MARFE develops, $n_{\text {MARFE }}$ and $T_{\text {MARFE }}$, are determined by the intersection of the curves $n_{c r}(T)$ and $n_{s t}(T)$.

\section{DISCUSSION OF RESULTS}

Figures 4(a) and 4(b) show the dependences $n_{c r}\left(T_{L}\right)$ and $n_{s t}\left(T_{L}\right)$ computed for deuterium auxiliary heated plasma, $q_{\text {core }}=4 \mathrm{~W} / \mathrm{cm}^{2}, \quad \kappa_{\perp}=2 \times 10^{20} \mathrm{~m}^{-1} \mathrm{~s}^{-1}$, in TEXTOR with carbonized and boronized walls, respectively. The solid curves were found according to the formulas and assump- tions in the preceding section. One can see that in both situations, $n_{\text {MARFE }}$ at the intersection points 1 is roughly the same, in disagreement with experimental observations. ${ }^{15}$ This can be explained by the fact that under conditions of fast hydrogen desorption from the wall, $\mu \gg \gamma$ and $\xi_{w} \approx 1$, impurity radiation is not the dominant trigger of MARFE. This becomes evident by considering the dashed curves, which give $n_{c r}$ computed without impurity contribution at all. Under these conditions, MARFE onset occurs at the point 2 and is purely due to "recycling" instability. ${ }^{53,54}$ By this instability, the convective energy loss acts instead of radiation. A spontaneous reduction of the temperature inhomogeneously on the magnetic surface leads through the pressure equilibration to an increase of the density. Therefore, the perpendicular particle and energy flow to the wall grows and leads to a further plasma cooling. Formally, this mechanism is represented by the recycling terms in $E_{*}$ proportional to $\phi_{T}$ and $\phi_{i}$ [see the line after Eq. (19)]. The relevance of "recycling" instability to MARFE formation was first discussed in Ref. 54.

The significant increase of $n_{\text {MARFE }}$ after wall boronization observed in the experiment ${ }^{15}$ can be interpreted by the change in the hydrogen recycling. It is generally reported (see, e.g., Refs. 19 and 44) that boronization results in a much more effective wall pumping then carbonization. This would mean that the desorption rate coefficient $\mu$, see Eq. (15) and Eq. (16), is significantly lower for boronized walls then for carbonized ones. With decreasing $\mu$ the coefficient $\xi_{w}$ decreases from its maximum value 1 , corresponding to a complete recycling, to a level of $R_{P}$ in the limit case with $\mu=0$. For the conditions in question, $R_{P} \approx 0.2^{50}$ and a significant reduction in the response of the hydrogen neutral influx and ion outflow to a temperature perturbation should be expected. As a result, the recycling contribution to the instability practically vanishes and the critical density noticeably increases. The $n_{c r}\left(T_{L}\right)$ computed for $\xi_{w}=0.4$ is shown in Fig. 4(b) by the dotted curve. In this case $n_{\text {MARFE }}$ at the point 3 is significantly higher than in the case of carbonized walls in agreement with experimental observations. The qualitative change of the MARFE threshold nature when going from carbonized to boronized walls, from predominantly driven by recycling instability to driven by impurity radiation, can be seen in the modification of the experimental scaling for the density limit. ${ }^{15}$

Finally, we demonstrate that the model proposed here for MARFE threshold explains also recent findings in JET. ${ }^{14}$ The HFS "wall" MARFE determined the density limit in $L$-mode deuterium JET plasmas, however, in helium discharges the "wall" MARFE did not occur and much higher plasma densities were achieved. Remarkably, this happened even though the impurity concentration in helium plasmas is slightly higher compared to that in deuterium plasmas of the same density due to a higher physical sputtering of carbon limiters by helium ions. However, in the vicinity of the sputtering threshold the erosion coefficient drops very fast with decreasing temperature and this provides a stabilizing effect. In addition, the ionization rate of helium atoms is also very sensitive to the electron temperature in the range in question, which additionally suppresses instability. Indeed, a reduction 
of the edge temperature leads to a deeper penetration of recycling helium neutrals and decrease of the plasma flux, $\Gamma_{p}$ (see $d \ln \sigma_{*} / d \ln T$ in the factors $\phi_{T}, \phi_{i}$, and $\phi_{\mathrm{rad}}$ ). This, first, amplifies the reduction of the impurity influx and, second, leads to a decrease of convective energy losses from the plasma, i.e., "recycling" instability can be suppressed. This is confirmed in Fig. 4(c) where $n_{c r}(T)$ and $n_{s t}(T)$ dependencies are demonstrated for JET helium plasmas with carbon as the dominant impurity $\left(R=3 m, q=4.5, q_{\text {core }}=3.5 \mathrm{~W} / \mathrm{cm}^{2}\right.$, $D_{\perp}=1 \mathrm{~m}^{2} / \mathrm{s}, \kappa_{\perp}=10^{20} \mathrm{~m}^{-1} \mathrm{~s}^{-1}, \delta_{T}=3 \mathrm{~cm}$ were assumed). There is no intersection of these curves and the plasma is stable with respect to the "wall" MARFE at any density.

Although we have confined our consideration only to the wall MARFE, the recycling instability can be also of importance for the development of X-point MARFE normally preceding the wall MARFE in divertor machines. Observations on many tokamaks ${ }^{1}$ indicate that the perpendicular particle fluxes responsible for this instability start to dominate the particle and heat transport in the scrape-off layer and at the edge close to the density limit. Recent modelling with the code 2D UEDGE show that these fluxes indeed can trigger $\mathrm{X}$-point MARFE ${ }^{55}$ With a proper modification of metric coefficients the present model can be applied to analyze this situation. However, even in their simplest form these coefficients are complex functions of the poloidal angle in the vicinity of the X-point(s). To date, this has not permitted a consistent analytical treatment of the problem. For example, a consideration for the X-point in Ref. 6 did not lead to any explicit formula for the MARFE threshold, even for a simplified case of isolated Fourier harmonics. For a more adequate treatment of the eigenfunction problem, a consistent numerical approach seems to be unavoidable. At the present time, we can only qualitatively state that similar to the case of the wall MARFE the position of the X-point MARFE is determined by the local maximum in the distance between neighboring magnetic surfaces and thus the weakest perpendicular heat transport. Normally, this maximum distance near the X-point is significantly larger than that at the high field side due to the Shafranov shift of magnetic surfaces and therefore the X-point MARFE develops earlier.

In the present paper, we have used the very primitive ansatz of perpendicular transport coefficients independent of plasma parameters. By introducing more sophisticated transport models, both $L$ - and $H$-mode conditions can be considered. Such a generalization of the model in future could lead to an important understanding of the nature of $H-L$ transition and of the density limit in general.

Finally, we note that the current approach allows one to speculate about the nature of phenomena observed in Large Helical Device (LHD) close to the density limit, ${ }^{56,57}$ where the development of a MARFE-like structure was preceded by a poloidally symmetric cooling of the edge plasma. The poloidal width of unstable perturbations is governed by the factor $p$ [see Eqs. (22) and Ref. 7]: the smaller $p$ the smoother is the perturbation on the magnetic surface. Although there is currently no formal generalization of this expression on a three-dimensional magnetic geometry like LHD, qualitatively one should use the connection length between inboard and outboard sides of the device instead of $q R$. Due to toroidal asymmetry, this length and, thus, $p$ are essentially smaller in stellarators than in tokamaks. Therefore one should expect more poloidally homogeneous unstable perturbations in stellarators. When the perturbation grows the plasma temperature and the parallel heat conductivity $\kappa_{\|}$ $\sim T^{2.5}$ drop and $p \sim 1 / \kappa_{\|}$increases leading to a more pronounced MARFE-like shape. A more quantitative consideration of this situation will be done elsewhere.

\section{CONCLUSION}

Experimental observations show that plasma-wall interaction plays a crucial role in the development of thermal instabilities at the edge. In the preceding analysis, this role was considered from several perspectives. First, an approach was provided, which estimates the radiation losses from locally released impurity, for which the transport effects are of principal importance and a noncorona model must be used. This approach permits the assessment of the nature of the impurity source and its dependence on plasma parameters. Second, the release of the working gas neutrals from the wall and the plasma outflow through the LCMS are incorporated into the model and related to the characteristics of the wall and neutrals themselves, e.g., their ionization rate. Therefore, the importance of these properties for the MARFE formation can be evaluated. In agreement with observations on TEXTOR, the model predicts a higher density limit for discharges in a machine with boronized walls compared to the same machine with carbonized walls. The model also predicts that a "wall" MARFE does not develop in helium discharges, as observed in JET.

\section{ACKNOWLEDGMENTS}

The authors would like to thank Dr. R. E. H. Clark, Dr. J. Abdallah, Jr., and Professor R. Janev for providing data on boron radiation.

${ }^{1}$ M. Greenwald, Plasma Phys. Controlled Fusion 44, R27 (2002).

${ }^{2}$ B. Lipschultz, B. LaBombard, E. S. Marmar, M. M. Pickrell, J. L. Terry, R. Watterson, and S. M. Wolfe, Nucl. Fusion 24, 977 (1984).

${ }^{3}$ J. F. Drake, Phys. Fluids 30, 2429 (1987).

${ }^{4}$ W. M. Stacey, Plasma Phys. Controlled Fusion 39, 1245 (1997).

${ }^{5}$ A. De Ploey, R. A. M. Van der Linden, G. T. A. Huysmans, M. Goossens, W. Kerner, and J. P. Goedbloed, Plasma Phys. Controlled Fusion 39, 423 (1997).

${ }^{6}$ Yu. Igithanov and M. Mikhailov, J. Nucl. Mater. 266-269, 837 (1999).

${ }^{7}$ M. Z. Tokar, Phys. Plasmas 8, 2866 (2001).

${ }^{8}$ J. L. Terry, E. S. Marmar, S. M. Wolfe et al., Bull. Am. Phys. Soc. 26, 886 (1981).

${ }^{9}$ F. Alladio, R. Bartiromo, B. Casali et al., Phys. Lett. 90A, 405 (1982).

${ }^{10}$ D. R. Baker, R. T. Snider, and M. Nagami, Nucl. Fusion 22, 807 (1982).

${ }^{11}$ J. Rapp, P. C. de Vries, F. C. Schüller et al., Nucl. Fusion 39, 765 (1999).

${ }^{12}$ J. Rapp, W. Fundamenski, A. Huber et al., in 28th EPS Conference on Controlled Fusion, Funchal, 2001, edited by C. Silva, C. Varandas, and D. Campbell (European Physical Society, Petit-Lancy, 2001), ECA Vol. 25A, p. 981.

${ }^{13}$ P. C. de Vries, J. Rapp, F. C. Schüller, and M. Z. Tokar, Phys. Rev. Lett. 80, 3519 (1998).

${ }^{14}$ J. Rapp, A. Huber, L. C. Ingesson, S. Jachmich, G. F. Matthews, V. Philipps, and R. Pitts, J. Nucl. Mater. 313, 524 (2003).

${ }^{15}$ J. Rapp, W. Biel, H. Gerhauser et al., J. Nucl. Mater. 290-293, 1148 (2001)

${ }^{16}$ W. Eckstein, J. Bohdansky, and J. Roth, Nucl. Fusion Suppl. 1, 51 (1991).

${ }^{17}$ P. Bogen and D. Rusbüldt, Nucl. Fusion 32, 1057 (1992). 
${ }^{18}$ A. A. Haasz, J. A. Stephens, E. Vietzke, W. Eckstein, J. W. Davis, and Y. Hirooka, Nucl. Fusion Suppl. 7A, 9 (1998).

${ }^{19}$ S. Higashijima, T. Sugie, H. Kubo et al., J. Nucl. Mater. 220-222, 375 (1995).

${ }^{20}$ D. E. Post, R. V. Jensen, C. B. Tarter, W. H. Grasberger, and W. A. Lokke, At. Data Nucl. Data Tables 20, 397 (1977).

${ }^{21}$ M. Z. Tokar, Plasma Phys. Controlled Fusion 36, 1819 (1994).

${ }^{22}$ T. Tazima, M. Tanaka, M. Yoshikawa, and K. Inoue, Nucl. Fusion 14, 517 (1974).

${ }^{23}$ Yu. N. Dnestrovskij, I. N. Inovenkov, and D. P. Kostomarov, Nucl. Fusion 16, 513 (1976).

${ }^{24}$ T. Amano and M. Okamoto, J. Phys. Soc. Jpn. 42, 1019 (1977).

${ }^{25}$ P. H. Rutherford, S. P. Hirshman, R. V. Jensen, D. E. Post, and F. G. P. Seidl, Proceedings of Papers, International Symposium on Plasma Wall Interaction, Jülich, 1976 (Pergamon, Oxford, 1977), p. 173.

${ }^{26}$ R. A. Hulse, Nucl. Technol./Fusion 3, 259 (1983).

${ }^{27}$ Yu. N. Dnestrovskij and G. V. Pereverev, Nucl. Fusion 23, 633 (1983)

${ }^{28}$ T. E. Stringer, 12th EPS Conference on Controlled Fusion and Plasma Physics, Budapest, 1985, edited by L. Pocs and A. Montvai (European Physical Society, Petit-Lancy, 1985), ECA Vol. 9F, part 1, p. 86.

${ }^{29}$ R. A. Hulse, D. E. Post, and D. R. Mikkelsen, J. Phys. B 13, 3895 (1980).

${ }^{30}$ S. L. Allen, M. E. Rensink, D. N. Hill et al., J. Nucl. Mater. 196-198, 804 (1992).

${ }^{31}$ D. E. Post, J. Nucl. Mater. 220-222, 143 (1995).

${ }^{32}$ M. Z. Tokar, Contrib. Plasma Phys. 28, 355 (1988).

${ }^{33}$ X. Bonnin, R. Marchand, and R. K. Janev, Nucl. Fusion Suppl. 2, 117 (1992).

${ }^{34}$ W. Engelhardt and W. Feneberg, J. Nucl. Mater. 76-77, 518 (1978).

${ }^{35}$ U. Samm, P. Bogen, H. A. Claassen, H. Gerhauser, H. Hartwig, E. Hintz, Y. T. Lie, A. Pospieszczyk, D. Rusbüldt, and B. Schweer, J. Nucl. Mater. 176-177, 273 (1990).

${ }^{36}$ G. Sergienko, A. Pospieszczyk, M. Lehnen, M. Brix, J. Rapp, B. Schweer, and P. T. Greenland, J. Nucl. Mater. 290-293, 720 (2001).

${ }^{37}$ F. A. Kelly, W. M. Stacey, J. Rapp, and M. Brix, Phys. Plasmas 8, 3382 (2001).
${ }^{38}$ P. C. Stangeby, The Plasma Boundary of Magnetic Fusion Devices (Institute of Physics, Bristol, 2000).

${ }^{39}$ M. Z. Tokar, W. Biel, J. Rapp, D. Reiser, U. Samm, and G. Sergienko, Contrib. Plasma Phys. 42, 290 (2002).

${ }^{40}$ M. Z. Tokar, Phys. Plasmas 7, 2432 (2000).

${ }^{41}$ M. Z. Tokar, Plasma Phys. Controlled Fusion 35, 1119 (1993).

${ }^{42}$ R. Janev, D. E. Post, W. D. Langer, K. Evans, D. B. Heifetz, and J. C. Weisheit, J. Nucl. Mater. 121, 10 (1984).

${ }^{43}$ U. Samm, P. Bogen, G. Esser et al., J. Nucl. Mater. 220-222, 25 (1995).

${ }^{44}$ J. Winter, H. G. Esser, L. Könen et al., J. Nucl. Mater. 162-164, 713 (1989).

${ }^{45}$ V. M. Sharapov, S. V. Mirnov, S. A. Grashin, S. V. Lebedev, I. A. Kogan, A. V. Krasilnikov, V. A. Krupin, L. S. Levin, A. N. Romannikov, and A. P. Zakharov, J. Nucl. Mater. 220-222, 730 (1995).

${ }^{46}$ F. L. Tabares, E. della Cal, D. Tafalla, R. Balbín, T. Estrada, I. GarsíaCortés, M. A. Ochando, M. A. Pedrosa, and B. Zurro, J. Nucl. Mater. 220-222, 688 (1995).

${ }^{47}$ M. L. Apicella, G. Mazzitelli, G. Apruzzese et al., J. Nucl. Mater. 313316, 269 (2003)

${ }^{48}$ C. H. Skinner, H. W. Kugel, R. Maingi et al., Nucl. Fusion 42, 329 (2002).

${ }^{49}$ G. Federici, C. H. Skinner, J. N. Brooks et al., Nucl. Fusion 41, 1967 (2001).

${ }^{50}$ G. M. McCracken and P. E. Stott, Nucl. Fusion 19, 898 (1979).

${ }^{51}$ M. Z. Tokar, Phys. Plasmas 9, 1646 (2002).

${ }^{52}$ Handbook of Mathematical Functions, 9th ed., edited by M. Abramowitz and I. A. Stegun (Dover, New York, 1970), p. 722.

${ }^{53}$ M. Z. Tokar, Phys. Scr. 31, 411 (1985).

${ }^{54}$ M. Z. Tokar, J. Rapp, D. Reiser, U. Samm, F. C. Schüller, G. Sergienko, and P. C. de Vries, J. Nucl. Mater. 266-269, 958 (1999).

${ }^{55}$ X. Q. Xu, W. M. Nevins, T. D. Rongnlien, R. H. Bulmer, M. Greenwald, A. Mahdavi, L. D. Pearlstein, and P. Snyder, Phys. Plasmas 10, 1773 (2003).

${ }^{56}$ B. J. Peterson, Y. Xu, S. Sudo et al., Phys. Plasmas 8, 3861 (2001).

${ }^{57}$ Y. Xu, B. J. Peterson, S. Sudo et al., Nucl. Fusion 42, 601 (2002). 